\title{
Ueber Wasserreinigung und Wasseranalysen.
}

E. Bohlig in Eisenach.

Im Archiv der Pharm. habe ich s. Zeit die chem. Processe, woraus sich eine neue Methode der Wasserreinigung begründet, näher besprochen und besonders darauf hingewiesen, dass bei gewöhnlicher Temperatur und bis zu $100^{\circ}$ Cels. basisch-kohlensaure Magnesia ausserordentlich leicht Gyps, Chlorcalcium etc. zersetzt, ebenso wie in höheren Hitzgraden umgekehrt Magnes. sulfat und kohlensaurer Kalk wieder Gyps und kohlens. Magnesia bildeten, wie in den Dampfkesseln.

Ich habe ferner angegeben, dass beide Processe gleich leicht und glatt verlaufen, so dass die Magnesia unstreitig das billigste und rationellste Wasserreinigungs-Mittel sei. Diese Methode, zn deren Gunsten die Praxis bereits entschieden hat, wurde gleich nach dem Bekanntgeben besonders von Dr. F. Fischer in Hannover angegriffen.

Die abfällige Kritik erging sich zunächst in dem Nachweise, dass meine Wasserreinigung nichts Neues biete, indem schon Mitscherlich erwähnte, dass Magnesia mit Gyps sich umsetze etc.

In meiner damaligen Entgegnung wies ich Fischer nach, dass diese Umsetzung nicht stattfinde, sondern sich nur auf gefällte kohlensaure Magnesia beziehe, diese Thatsache aber bereits um 1780 angegeben werde.

Die Priorität muss somit Bergmann zuerkannt werden.

Wohl aber nehme ich für mich in Anspruch, der erste gewesen zu sein, welcher die vollständige Consequenz dieses Processes gezogen und in Folge dessen die grosse Bedeutung desselben im Naturhaushalt bestimmt nachgewiesen hat.

Fischer selbst sagt in seinem neuesten Werke: Chem. Technologie des Wassers 1878. Erste Lieferung. pag. 132: „Oft, namentlich bei Lntersuchung der Kesselspeisewasser, ist es erforderlich, diejenige Menge von Kalk und Magnesia zu bestimmen, welche als Bicarbonat vorhanden ist und durch längeres Kochen als einfacb kohlensaure Verbindungen ausgeschieden wird etc. 
Fischer ibersieht hierbei, dass bei den zeitherigen Analysen und Auskochen der Wässer - nur kohlensaure Magnesia - in schwefelsaure, salzsaure und salpetersaure Magnesia überführt wird.

Die nachfolgenden Mittheilungen, Ergebnisse meiner zahlreichen Untersuchungen in dieser Frage dürften desshalb auch genügendes wissenschaftliches Interesse bieten; dieselben beziehen sich

1) auf das Verhalten des kohlensauren Kalks in Kohlensäure haltendem Wasser (Nichtexistenz des sog. Calciumbicarbonats),

2) auf das Verhalten der kohlensauren Magnesia in Lösungen von Gyps, Chlorcalcium etc., überhaupt auf die wichtige Rolle, welche das verkannte Magnesiumcarbonat in den verschiedenen natürlichen Wässern spielt,

3) auf die Zusammenstellung der Wasseranalysen, entsprechend der wirklichen Zusammensetzung der Mineralwässer.

Es wurden zunächst Lösungen hergestellt von reinem kohlensaurem Kalk in mit Kohlensäure bei gewöhnlichem Druck und Temperatur gesättigtem destillirt. Wasser. Der Maximalgehalt war $0,12 \mathrm{~g}$. $\mathrm{CaCO}^{3}$ pro $100 \mathrm{C}$. C. Wasser; dabei kamen auf 1 Aequivalent $\mathrm{Ca} \mathrm{CO}^{3}$ über 6 Aequiv. Kohlensäure. Betrachten wir diese Lösungen (in unverschlossenen Gefässen an freier Luft stehend) durch fortlaufende Untersuchungen des Gehaltes an Säure und Basis $\left(\mathrm{CaCO}^{3}\right)$, so zeigt sich folgendes interessante Verhalten.

Die Untersuchung begann nach 6 stündigem Stehen stets auf 100 C.C. Flüssigkeit bezogen:

$$
\begin{aligned}
& \text { I. Tag }\left\{\begin{array}{ll}
0,073 & \text { g. } \mathrm{CaCO}^{3} \\
0,0915-\mathrm{CO}^{2}
\end{array}\right\} \text { oder } \begin{cases}1 & \text { Aequiv. } \\
2,9- & \mathrm{CaCO}^{3}\end{cases}
\end{aligned}
$$

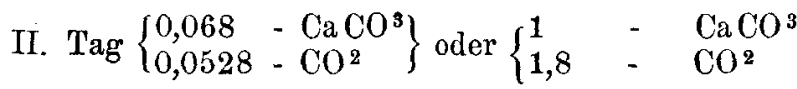

$$
\begin{aligned}
& \text { III. Tag }\left\{\begin{array}{ll}
0,056 & -\mathrm{CaCO}^{3} \\
0,044 & -\mathrm{CO}^{2}
\end{array}\right\} \text { oder }\left\{\begin{array}{lll}
1 & - & \mathrm{CaCO}^{3} \\
1,8 & - & \mathrm{CO}^{2}
\end{array}\right.
\end{aligned}
$$




$$
\begin{aligned}
& \text { IV. Tag }\left\{\begin{array}{ll}
0,040 & \text { g. } \mathrm{CaCO}^{3} \\
0,026 & -\mathrm{CO}^{2}
\end{array}\right\} \text { oder }\left\{\begin{array}{lll}
1 & \text { Aequiv. } \\
1,5-\mathrm{Ca}^{3} & \mathrm{CO}^{2}
\end{array}\right. \\
& \text { V. Tag }\left\{\begin{array}{ll}
0,028-\mathrm{CaCO}^{3} \\
0,0219-\mathrm{CO}^{2}
\end{array}\right\} \text { oder }\left\{\begin{array}{lll}
1 & - & \mathrm{CaCO}^{3} \\
1,7 & - & \mathrm{CO}^{2}
\end{array}\right. \\
& \text { XIV. Tag }\left\{\begin{array}{ll}
0,008-\mathrm{CaCO}^{3} \\
0,0105-\mathrm{CO}^{2}
\end{array}\right\} \text { oder }\left\{\begin{array}{lll}
1,0 & - & \mathrm{CaCO}^{3} \\
1,8 & - & \mathrm{CO}^{2}
\end{array}\right.
\end{aligned}
$$

Es giebt somit keinen Punkt, wo auch nur vorübergehend auf 1 Aeq. Monocarbonat 1 Aeq. Kohlensäure kommen; es scheidet sich vielmehr weit über der Grenze des Bicarbonats schon Monocarbonat aus, so dass in der Lösung von ein em Aequivalent dieses Salzes mindestens bis 11/2 Aequiv. Kohlensäure verbleiben.

Noch deutlicher tritt diese Erscheinung hervor, wenn man einer solchen Lösung von Monocarbonat in überschüssiger Kohlensäure genau so viel Kalkwasser beimischt, dass im Gemisch nun blos Bicarbonat enthalten sein müsste. Eine alsbaldige Ausscheidung von einfach kohlensaurem Kalk zeigt ganz denselben Vorgang; die Lösung enthält nur noch geringe Mengen einfach kohlensauren Kalk mit $1 \frac{1}{2}-2$ Aequiv. freier Kohlensäure.

Nach sehr langem Stehen dieser Lösungen an der Luft tritt (wie oben in der Tabelle beim XIV. Tag) ein Punkt ein, wo das Auskrystallisiren des Calciummonocarbonats still steht. Es entspricht dies offenbar der Grenze, wo die Löslichkeit des einfach kohlensauren Kalks im Wasser an sich beginnt, und zwar noch vermehrt um ein Geringes durch diejenige kleine Menge freier Kohlensäure, welche reines Wasser nach langem Stehen der Luft entnimmt und welche unter denselben Umständen folglich nicht aus dem Wasser verdampfen kann.

Es wurden ferner - bei gewöhnlichem Druck und Temperatur gesättigte Lösungen von Calciumcarbonat in Kohlensäure - successive erwärmt und bei $10,20,40,60,80$ u. $100^{\circ}$ Cels. untersucht. Der Hergang ist, wie ich erwartete, in allen Theilen derselbe; der Siedepunkt entspricht sogar ziemlich genau jenem bei langem Stehen in der Kälte erreicht werdenden stabilen Gehalt von $0,006-8 \mathrm{~g}$. $\mathrm{CaCO}^{3}$ (in $100 \mathrm{C.C}$ ) auf wiederum bis $1 \frac{1}{2}$ Aequiv. freier Kohlensäure. 
Derselben Behandlung unterwarf ich Lösungen von Calciumcarbonat in $\mathrm{CO}^{2}$ halt. Wasser, welchen beigemischt waren

a) äquivalente Mengen Magnesiumsulfat oder Chlorid,

b) - - Calciumsulfat

c) alle diese Verbindungen, wie sie in natürlichen Wässern vorkommen.

Statt die einzelnen Versuche speciell aufzuführen, welche sowohl beim Stehen in der Kälte, als auch bei successivem Erwärmen durchaus dieselben Ergebnisse lieferten, sei nur folgender besonders hervorgehoben.

Eine reine Lösung von Magnes. Carbonat:

100 C.C. sättigten genau 31,2 C.C. $1 / 10$ Norm.-Säure, ferner :

100 C. C. absorbirten Kalkwasser entsprechend $=70,4$ C.C. $1 / 10$ Säure.

100 C.C. dieser kohlens. Magnes. - Lösung wurden mit so viel Kalkwasser versetzt, dass in der resultirenden Lösung genau auf 1 Aequiv. $\mathrm{MgO}=1$ Aeq. Kohlensäure entfielen.

Nach Hinzufügen von überschüssigem Gyps und Chlorcalcium wurde einige Male aufgeschüttelt und wohl verschlossen klären gelassen.

100 C.C. der klaren Lösung erforderten zur Sättigung 0,6 C.C. $1 / 10$ Säure, entsprechend nur noch einen Gehalt von 0,006 g. $\mathrm{Ca} \mathrm{CO}^{3}$, also dasselbe Resultat wie bei dem analogen Versuch mit Calciumcarbonatlösung behuf's gänzlicher Ausfällung des Kalkes, nur mit dem Unterschied, dass bei letzterem Versuch schon die Wegnahme der Kohlensäure bis zum sogenannten Bicarbonat hin genügt, während Magnesiumcarbonat die Kalksalze zu fällen beginnt, wenn die Kohlensäure durch irgend welche Mittel bis zum Monocarbonat weggenommen wird.

Aus diesen Versuchen geht zunächst hervor, dass die Annahme eines Calciumbicarbonates durch nichts gerechtfertigt ist. Ein solches im chemischen Sinne existirt nicht.

Ferner, dass das Magnesmonocarbonat schon sämmtliche Kalksalze sofort zersetzt und einfach kohlensauren Kalk ausscheidet und dass diese Zersetzung schon soweit geht, dass nur noch Spuren Kalk gelöst bleiben.

Arch. d. Pharm. XIII. Bds, 1. Heft. 
Es liegt nun gewiss sehr nahe, dass die natürlichen Wässer sich genau ebenso verhalten werden, wenn man sich zur Wegnahme der freien transcarbonaten Kohlensäure geeigneter Mittel bedient. Dieses geeignete Mittel fand ich in dem Magnesiumoxydhydrat.

Versetzt man ein natürliches Wasser in sicherem Ueberschuss damit, schüttelt einige Male gut durch und lässt klären, so ist das Filtrat so $\mathrm{kalkfrei}$, dass oxalsaur. Anmon. nach langem Stehen kaum eine Reaction giebt; das $\mathrm{Was}$ ser istalso in der That schon in der Kälte vollk ommen genügend gereinigt. Eine Ausnahme machen natïrlich nur jene $W$ ässer, welche neben grossen Mengen Gyps etc. wenig Erdcarbonate enthalten, wo es also an Kohlensäure fehlt und die man in Form von Feuerluft leicht hinzufügen kann. (Weit aus der grösste Theil aller Quell und Bachwässer enthält aber genügende Mengen eigener Kohlensäure).

Das geglühte Magnesiumoxyd ist an sich ein ziemlich inerter Körper, der nur nach tagelanger Berührung mit Wasser, dann aber vollständig, in Magnesoxydhydrat übergeht.

Da nun in der Praxis diese Hydratbildung in bester Weise stillschweigend vor sich geht, in chem. Laboratorien (wie wiederholt bei Ferd. Fischer) aber unbegreiflicher Weise anch dann noch vernachlässigt wird, nachdem ich diese vorhergehende Hydratbildung zur Conditio sine qua non gemacht, so habe ich gewiss ein Recht, dergl. Versuche als nichts beweisend zu bezeichnen und günstigere Resultate zu prophezeien bei Anwendung von geglïhtem Magnesiumoxyd-Pulver erst, nachdem dasselbe einige Tage unter Wasser gestanden hat.

Ich habe ferner behouptet, dass man über die Zusammensetzung der natürlichen Wasser vielfach falscher Ansicht sei und muss dies näher begründen.

Meine obigen Versuche haben dargethan, dass Calciumbicarbonat gar nicht existiren kann, sondern, dass das dafür Ausgegebene - Lösungen von Monocarbonat sind in stets mehr Kohlensäure, als dem sog. Bicarbonat entspricht. 
Folgerichtig müssen auch alle natürlichen Kalkwässer mehr oder weniger freie Kohlensäure besitzen, während nach der zeitherigen Annahme dies nicht der Fall sein soll. Brunnenwässer , sollen nur ganz selten freie Kohlensäure haben.“

Thatsache ist, dass eine ganze Menge Brunnenwässer mehr Kohlensäure haben, als dem reinen Bicarbonat entsprechen würde und dass dieser KohlensäureGehalt in Beziehung zur Basis ein zufälliger und schwankender ist, was nach obigen Versuchen ganz begreiflich erscheint.

Es würde im Gegentheil schwer einzusehen sein, warum das - zufällige Gemisch - von 1 Aequiv. $\mathrm{CaCO}^{3}$ und 1 Aeq. $\mathrm{CO}^{2}$ die Regel bilden sollte.

Eine ganze weitere Reihe von Brunnenwässern enthalten nun aber in der That kaum mehr Kohlensäure, als sie dem Bicarbonat entsprechen würde und wieder andere sogar nur wenig mehr, als dem Monocarbonat entspricht.

Auch hierfür liegt die Erklärung nicht fern; nachdem wir gesehen haben, dass Magnesiumcarbonat erst dann die Kalksalze zersetzt, wenn durch irgend welche Mittel die freie (transcarbonate) Kohlensäure weggenommen wurde.

Wenn also natürliche $W$ ässer diesen geringen Kohlensäure-Gehalt der Basis gegenüber zeigen, so müssen solche Wässer nicht nur magnesiahaltig sein, sondern es muss so viel Magnesia wenigstens vorhanden sein, dass dieselbe die ganze Basicität des betreffenden Wassers deckt, mit andern Worten: in solchen Wässern ist nur die eine Annahme zulässig, dass nur kohlensaure Magnesia darin vorkommt und aller Kalk an stärkere Säuren gebunden sein muss.

Die geringe Zahl dieser von mir bis jetzt untersuchten Wässer verhält sich in der That so; es ist soviel Magnesia vorhanden, wie dem Abstumpfungsgrade der Wässer (mit der Normalsäure) entspricht; meistens darüber.

Da die zeitherige entgegengesetzte Annahme nur Folge eines ganz falschen Analysirens war (Kochen der Wässer), so bedarf sie keiner besonderen Berücksichtigung, immerhin haben aber weitere Bestätigungen dieser Vorgänge zu 
geschehen und hat sich zu zeigen, ob meine Ansicht über die Sache die richtige ist.

1) Um nicht falsch verstanden zu werden, resumire ich dieselbe in Kürze dahin, dass reine Kalkwässer ohne mehr als nur Spuren von Magnesia auf $1 \mathrm{Aeq} . \mathrm{Ca} \mathrm{CO}^{3}$ stets mehr als 1 Aeq. Kohlensäure haben müssen; Ausnahmen können nur Wasser bilden, bei denen der Gehalt an Carbonat ein höchst geringer ist.

2) In gemischten Magnesia-Wässern kann der Kohlensäure-Gehalt soweit zurückgehen, dass auf 1 Aequiv. Monocarbonat wenig mehr als $1 / 2$ Aequiv. Kohlensäure kommt. Punkt 2 involvirt, wie leicht einzusehen, eine weitere Thatsache, welche eine klare Einsicht gewährt über die Zusammensetzung aller natürlichen Wässer nicht nur, sondern wichtige Schlüsse zulässt über deren Ursprung oder den Grad der bereits eingetretenen Degenerirung.

Was die Zusammensetzung der Wässer zunächst betrifft, so spricht Alles dafür, dass die Annahme von Calciumcarbonat (in überschüssiger Kohlensäure gelöst) neben Magnesiasalzen der stärkercn Säuren, besonders Magnesiumsulfat, überhaupt unzulässig ist. Die ganze Magnesia ist vielmehr als Carbonat vorhanden, und nur diejenige Menge derselben, welche die gefundene Basicität des Wassers überschreitet, kann als Magnesia-sulfat-chlorid oder nitrat bestehen.

Es wird Niemand mehrfach kohlens. Natron neben Gyps in einer Lösung annehmen können; hier theilt man ohne Weiteres der stärkeren Säure eine stärkere Basis zu. Dass aber Calcium, wenn auch nicht in demselben Grade, eine stärkere Basis ist als Magnesia, will man ja schon mit ihrer Stellung in der electrischen Spannungs - Reihe andeuten, ferner spricht dafür, dass sich, wie ich nachgewiesen habe, schon in einer Wärme ron ca. $130^{\circ}$ C. (im Kesselwasser) Bittersalz und Calciumcarbonat sofort umsetzen in Gyps und kohlensaur. Magnesia. Da als Grund hierfür nicht die Bildung eines unlöslichen Salzes angenommen werden kann, (denn der ursprüngliche einfach kohlens. Kalk ist im Kesselwasser unlös- 
licher als der neugebildete Gyps) - so ist es eben dic grössere Verwandtschaft, welche den Ausschlag giebt.

Zur endgültigen Entscheidung der angeregten Fragen ist vor Allem eine rasch ausfübrbare Methode nothwendig, welche neben dor erforderlichen Genauigkeit namentlich die Beziehungen der hier in Betracht kommenden Verbindungen möglichst wahrheitsgetreu giebt, auch wenn man, wie in vielen Fällen, nur kleine Wassermengen zur Verfügung hat.

Ich fuihre diese Analysen in folgender Weise aus, wobei sich jede Bestimmun: auf ein und dieselbe Normalsalzsäure bezieht.

1) 100 C.C. des betreffenden Wassers werden mit Rothholztinctur versetzt, gekocht und tropfenweise mit 1/10 Normalsäure austitrirt. Der Farben-Uebergang aus dem tief Violetten ins Citronengelbe ist äusserst präcis bei $1 / 20$ C.C.

2) 100 C.C. kochend mit Ammoniak und oxalsaur. Ammon. ausgefällt, filtrirt, gewaschen und das Filterchen mit Inhalt geglüht im Platintiegel; alsdann in ein Kölbchen ohne Verlust gespiult und mit genau abgemessener Menge Normal-Salzsäure längere Zeit gekocht, bis vollständige Lösung erfolgt. Die Lösung mit Rothholztinctur versetzt und mit genau eingestellter Normal - Natronlösung tropfenweise versetzt bis zum Farben-Umschlag ins Violette. Man lässt die violette Flüssigkeit nochmals aufkochen und versetzt mit 1 Tropfon der Normalsäurc. - Die genommene Salzsäure giebt nach Abzug des Natrontitres und event. des Durchschnitts-Titres der Filterasche - den ganzen Kalk.

3) 100 C.C. Wasser werden mit 100 C.C. Kalkwasser versetzt, geschüttelt und verschlossen (event. noch mit Chlorcalcium) 6-8 Stunden stehen gelassen. Nach dieser Zeit 50 C. C. abpipettirt und filtrirt. Man erfährt so die verschluckten Kalkmengen - ausgedrückt in C.C. $1 / 10$ Säure. Die restirenden 150 C. C. werden mit einer Messerspitze voll Chlorammon versetzt, verschlossen, einige Mal tüchtig durchgeschüttelt und klären gelassen. Eine wiederholte Filtration ergiebt die neben den Monocarbonaten vorhandene freie Kohlen- 
säure. Die Differenz beider Titrationen liefert den Total - Magnesiagehalt.

Wasseranalysen.

\begin{tabular}{c|c|c|c|c}
\hline $\begin{array}{c}\text { No. } \\
\text { des } \\
\text { Was } \\
\text { sers. }\end{array}$ & $\begin{array}{c}\text { C. C. Zahl } \\
\text { 1/10 Säure zur } \\
\text { Sättigung der } \\
\text { Erden. }\end{array}$ & $\begin{array}{c}\text { C. C. Zabl } \\
\text { 1'10 Säure ent- } \\
\text { sprechend dem } \\
\text { ganzen Kalk. }\end{array}$ & $\begin{array}{c}\text { C. C. Zahl } \\
\text { 1/10 Säure für } \\
\text { Kohlensäure. }\end{array}$ & $\begin{array}{c}\text { C. C. Zahl } \\
\text { für } \\
\text { Totalmagnes. }\end{array}$ \\
\hline I. & 1,8 & 4,1 & 3,2 & 0,8 \\
II. & 1,0 & 3,8 & 2,0 & 0,9 \\
III. & 3 & 5,2 & 2,6 & 0,6 \\
IV. & 2,6 & 4,8 & 0,4 & 9,6 \\
V. & 2,4 & 9,0 & 0,4 & 8,8 \\
VI. & 4,4 & 8,6 & 3,6 & 13,6
\end{tabular}

Bei Wasser No I. ersehen wir, dass auf 1,8 Aeq. einfach kohlens. Erden 3,2 Aequiv. freie Kohlensäure kommen.

Die ganze Magnesia ist 0,8, somit als Carbonat zu berechnen und es bleiben für vorhandenes Calciumcarbonat nur $1 \mathrm{C}$. C.

Vom ganzen Kalk 4,1 kommen demnach 1 auf kohlensauren Kalk und 3,1 auf Gyps etc.

Die Analyse würde dann heissen:

$$
\begin{aligned}
& \text { \& } 1 \times 0,010 \text { g. }=0,010 \text { g. kohlensaurer Kalk. } \\
& \text { 总 } \quad 0,8 \times 0,0084-=0,0067 \text { - kohlensaure Magnesia. } \\
& \text { 일 } 3,1 \times 0,0136-=0,0421-\text { Gyps. } \\
& \text { 范 } 3,2 \times 0,0044-=0,0140 \text { - Kohlensäure. }
\end{aligned}
$$

Die Analyse des Wassers No. VI. (Wasser aus dem südlichen Russland) zeigt, dass auf 4,4 Aequiv. kohlens. Basis nur 3,6 Aeq. freie Kohlensäure kommen, dass aber viel mehr Magnes. $(13,6)$ vorbanden ist, als zur Sättigung von 4,4 C.C. Säure gehört. Das Wasser kann demnach keinen kohlensauren Kalk enthalten sondern nur kohlens. Magnes., somit:

$$
\begin{aligned}
4,4 \times 0,0084 & =0,0369 \mathrm{~g} . \text { kohlens. Magnesia. } \\
8,6 \times 0,0056 & =0,0481-\mathrm{CaO} \text { an } \mathrm{SO}^{3} \text { ? gebund. } \\
13,6-4,4 \times 0,0120 & =0,110-\mathrm{MgSO} \text {. } \\
3,6 \times 0,0044 & =0,0158 \cdot \text { freie Kohlensäure. }
\end{aligned}
$$

Dass man erst mit dieser richtigeren Ansicht über die Zusammensetzung der Speisewässer und nach einer genauen Analyse der freien Kohlensäure, der kohlens. Magnes. etc. 
meine Wasserreinigungsmethode richtig zu beurtheilen vermag, ist leicht einzusehen.

So ist klar, dass Wasser No. I. nur einen Zusatz von 3,2 Aequival. Magnesoxydhydr. bedarf, um dann 4 Aequivalente einfach kohlensaure Magnesia zu besitzen zur Ausfällung von ebenso viel Kalksalzen (Gyps), wovon hier nur 3,1 Aequivalente vorhanden sind. Dieses Wasser wird denn auch nach dem Umschütteln mit überschüssigem Magnesiumoxydhydrat sofort vollkommen frei von Kalk etc.

\title{
Mitthellungen zur Gesundheitspflege.
}

\author{
Von E. Reichardt.
}

\section{Mittel zur Vergiftung der Feldmäuse.}

Von behördlicher Seite wurde mir, als auch in das Feld der Gesundheitspflege einschlagend, die Frage zur Begutachtung unterbreitet, welche Mittel und Formen die geeignetsten seien, um die oft als Land - oder Feldplage auftretenden Mengen von Mäusen zu tödten. Die Ursache hierzu gab das örtlich erlassene Verbot vergifteter Körner wegen der grossen Verbreitung körnerfressender, nützlicher Thiere, obgleich hier stets gleichzeitig die Verordnung gilt, alle derartigen Gegenmittel nur in die Löcher der Mäuse einzufiullen, ein offenes Aufstreuen dagegen sogar mit Strafe belegt wird.

Bei Erwägung der Sachlage tritt zuerst die Frage auf, ob es nicht als gemeinschädlich zu bezeichnen und zu behandeln sei, wenn solche Mengen starker Gifte der Oeffentlichkeit überliefert werden, während man sonst mit der Austheilung von dergleichen schädlichen Stoffen so vorsichtig sich zeigt. Sind keine anderen Mittel und Wege geboten, um diesen Uebelständen entgegen zu treten! Ein gerechter Vorwurf triftt hierbei den Menschen selbst. Er vertilgt schonungslos, oft nur scheinbaren Vortheiles wegen oder auch nur aus Abneigung, die natürlichen Feinde solchen Ungeziefers. Aus Abneigung werden Schlangen, selbst die unschäd- 